\title{
Time-Motion Analysis of Young Competitive Surfers: Southern Portugal
}

Beatriz Minghelli*, Sara Graça, Sara Paulino and Inês Sousa

School of Health Jean Piaget Algarve, Piaget Institute, Portugal

*Corresponding author: Beatriz Minghelli, School of Health Jean Piaget Algarve, Piaget Institute, Enxerim, Silves, $8300-025$, Portugal, Tel: 282 440 170; E-mail: beatriz.minghelli@silves.ipiaget.pt

Received date: June 09, 2017; Accepted date: August 10, 2017; Published date: August 17, 2017

Copyright: $\odot 2017$ Minghelli B, et al. This is an open-access article distributed under the terms of the Creative Commons Attribution License, which permits unrestricted use, distribution and reproduction in any medium, provided the original author and source are credited.

\section{Abstract}

Introduction: Time-motion analysis should be an integral part of the coaching process in competitive surf to help design training programs, as well as maximize athlete performance. There are a few studies that analyzed the timemotion of surfer activities during competition. Thus, the aim of this study was evaluate each activity's time made by surfers during the heat competition, in order to account for the time spent in each surf activity.

Methods: Forty-two young Portuguese surfers, both males and females, residing in southern Portugal, participated in the championship of the "School Sport" of the Algarve region. Each surfer was individually videoed for the entire duration of the heat using a video camera. The V-Note Video Analysis Software 2.1.2 was used to evaluate the movement's time made by each surfer during the competition, in order to account for the time spent in each activity. The activities that the surfers were evaluated included: paddling; sprint paddle for wave; stationary; wave riding; miscellaneous (e.g. duck diving, recovery of the board, and other).

Results: The surfers were paddling $58 \%$, stationary $42 \%$, sprint paddle for wave $3.8 \%$, and wave riding $3.1 \%$ of the total time. The average of surfing activities were 15.2 seconds for paddling, 1.5 seconds to sprint paddle for wave, 8.8 seconds for stationary period, 4.4 for wave riding, and18.2 for miscellaneous activities.

Conclusion: The data from this study revealed that, for this sample and competition analyzed, the activities that had a greater percentage of time included the paddling and miscellaneous activities. The results of this study show that surfing is an intermittent activity that is characterized by large variability of each activity that was analyzed, and these surfing activities seems to be controlled more by environmental factors than by surfer attitude.

Keywords: Time-motion analysis; Surf; Competition

\section{Introduction}

Surfing is become more popular sport practiced at competitive and recreational levels on beaches worldwide [1], and the number of athletes in competitive surfing has also risen $[2,3]$. According to the International Surf Association [2], there are 35 million surfers in over 70 countries. The Portuguese Surf Federation [4] reports that there about 10,000 federated athletes, 70 clubs and 140 surf schools in Portugal. At a competitive level, surfing requires various high intensity physical capacities, such as muscular strength and endurance, balance, postural control and neuromuscular coordination [5]; in addition surfing involves intermittent high-intensity bouts of all out paddling, to gain enough momentum for the wave take-off, interspersed with short recovery periods and repeated bouts of low-intensity paddling [6,7]. Besides of that, surfing is characterized by intermittent bouts of exercise with variation of intensity and duration that requires high muscular endurance, moderate-high cardio-respiratory endurance and recovery, and anaerobic power of the upper torso [8].

To prescribe a training program for surfers it is necessary to take into account the duration of activity and the metabolism that is requested. Many factors can affect durations spent in each surfing activity, and its consequent intensity, such as ocean currents, wind orientation, type of sea floor, size of the waves, water temperature [5], type of wave break, frequency of waves, tides, and geographic location
$[7,8]$. Time-motion analysis should be an integral part of the coaching process in competitive surf to help design training programs, as well as maximize athlete performance, since the use of these technologies provide an opportunity to provide detailed and meaningful insights into surf and athlete performances [8]. Time-motion analysis refers to the frame-by-frame examination of video footage of each surfer during training or competition which registers the recording of time-anddistance data, movement patterns, frequency, mean duration and total time spent in activities [8].

To date there have been only two previous studies investigating the time-motion analysis of surfers during competition [1,6], and one study that investigated during training surf [9], and more one study in recreation surf [10]. Since the conditions of surfing are also dependent on the geographical location, it is important to carry out a timemotion analysis in the Portuguese surfers, since there is no study carried out in Portugal, and international studies are still scarce. Thus, the aim of this study was evaluate each activities time made by surfers during the heat competition, in order to account for the time spent in each surf activity.

\section{Materials and Methods}

A cross-sectional descriptive survey was used to collect data in this study. 


\section{Subjects}

The study population comprised Portuguese surfers, both males and females, residing in southern Portugal, who competed in last stage of the surf championship of the "School Sport" of the Algarve region on May 8, 2017.

Forty-two surfers participated in this championship (in Arrifana beach) distributed in the following categories: infants (age of birth: from 2004 until 2008), initiates (2002-2003), juveniles (1999-2001) and juniors (1995-1998).

This study was approved by the Regional School Sports Coordination.

\section{Heat analysis}

Each surfer was individually videoed for the entire duration of the heat using a video camera. All video recordings were filmed by four evaluators, positioned always in the same place, above sea level, in order to have the best view of the athletes in the water and minimize the periods when the wave passes through the athlete.

The duration of the heats was 15 minutes. In each heat, 4 surfers competed against each other, and the 2 best-scored surfers passed on to the next heat. The competition took place with 2 heats simultaneously. By random selection only the surfers who competed in the right heat were filmed.

Initially all surfers of the same heat were filmed, and each evaluator was responsible by filming the surfer who presented the same lycra Tshirt color in the various heats. For example, the evaluator 1 filmed only the surfers who competed with the red lycra. The end of filming of each evaluator took into account the battery time left of the video cameras.

The V-Note Video Analysis Software 2.1.2 was used to evaluate the movement's time made by each surfer during the competition, in order to account for the time spent in each activity.
The activities that the surfers were evaluated included:

Paddling: Alternating movements of the upper limbs towards the outside or in search of the best wave peak.

Sprint paddle for wave: Movement that the surfer performed in order to catch the wave, regardless of whether or not he can achieve that goal.

Stationary: Situation where the surfer could be positioned in the prone position or sitting on his board, without any type of locomotion activity.

Wave riding: It included the time of the take-off until the moment that the athlete lost contact of the feet with the board.

Miscellaneous: They included the duck diving, the recovery of the board after falling, getting out and entering in the water, when the image capture of the surfer was lost due to the passage of a wave, and other activities.

The time pattern of each surfer activity was analyzed, including the total and average time used to carry out each activity, the frequency and percentage of occurrence of each activity. All analysis was performed by a same evaluator.

\section{Data analysis}

Descriptive statistics were obtained regarding various surfing activities (paddling, sprint paddle for wave, stationary, wave riding and miscellaneous), including average, standard deviations, range and total duration.

The statistical analysis was performed with the Statistical Package for Social Sciences (SPSS), version 23.0.

\section{Results}

Sixteen surfers were filmed, corresponding to $39 \%$ of the study population (Table 1).

\begin{tabular}{|c|c|c|c|c|c|}
\hline Surfing activities & Paddling & Sprint paddle for wave & Stationary & Wave riding & Miscellaneous \\
\hline Average & $00: 13.3$ & $00: 03.4$ & $00: 07.4$ & $00: 06.7$ & $0: 07.8$ \\
\hline Standard deviation & $00: 15.2$ & $00: 01.5$ & $00: 08.8$ & $00: 04.4$ & $00: 18.2$ \\
\hline Range & $00: 00.6-01: 42.8$ & $00: 0.1 .1-00: 10.8$ & $00: 00.2$ - 0:13.2 & $00: 00.9-00: 16.4$ & $00: 00.2-04: 37.7$ \\
\hline Total time \% & $57.46 \%$ & $3.80 \%$ & $12.93 \%$ & $3.08 \%$ & $22.73 \%$ \\
\hline
\end{tabular}

Table 1: Time duration (minutes:seconds) of surfing activities.

Figures 1 to 5 show the time intervals (in seconds) of each surfing activity filmed during the competition (paddling, sprint paddle for wave, stationary, wave riding and miscellaneous). 


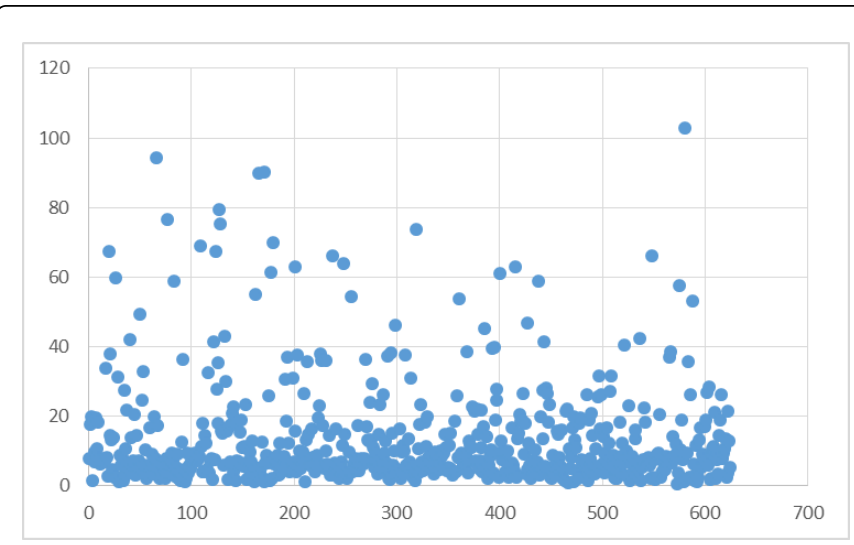

Figure 1: Number of paddling bouts (in seconds) performed during a competitive surfing heat (average \pm standard deviation).

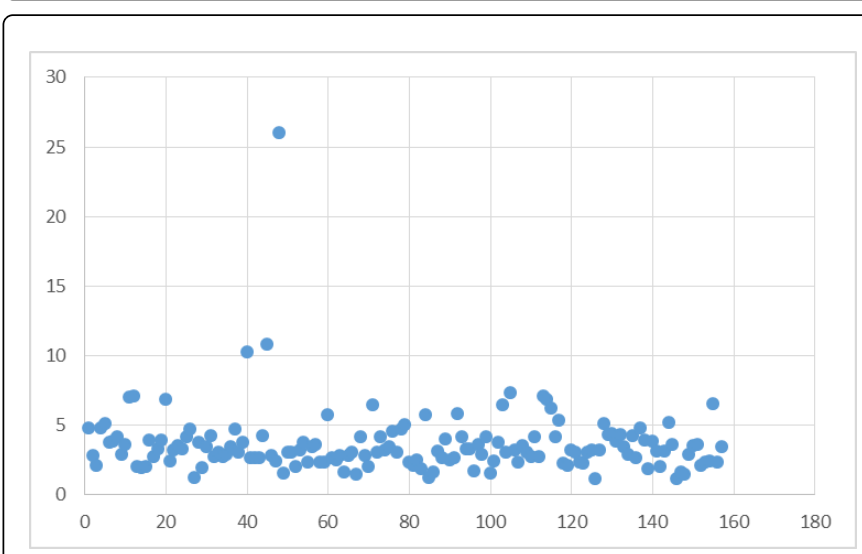

Figure 2: Number of sprint paddle for wave bouts (in seconds) performed during a competitive surfing heat (average \pm standard deviation).

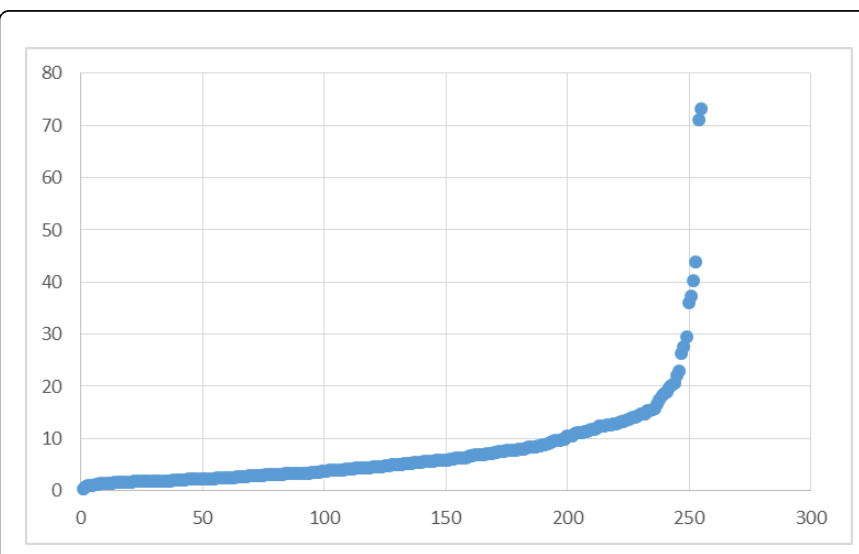

Figure 3: Number of stationary period bouts (in seconds) performed during a competitive surfing heat (average \pm standard deviation).

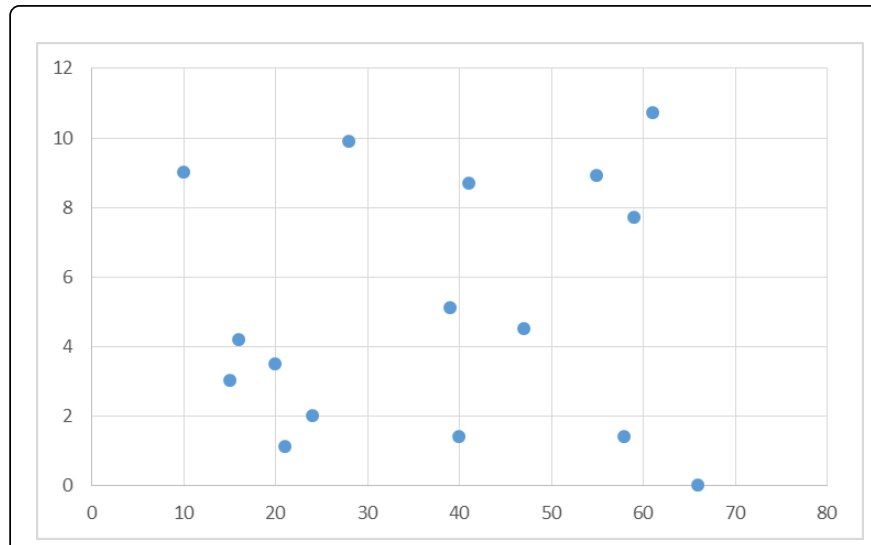

Figure 4: Number of wave riding bouts (in seconds) performed during a competitive surfing heat (average \pm standard deviation).

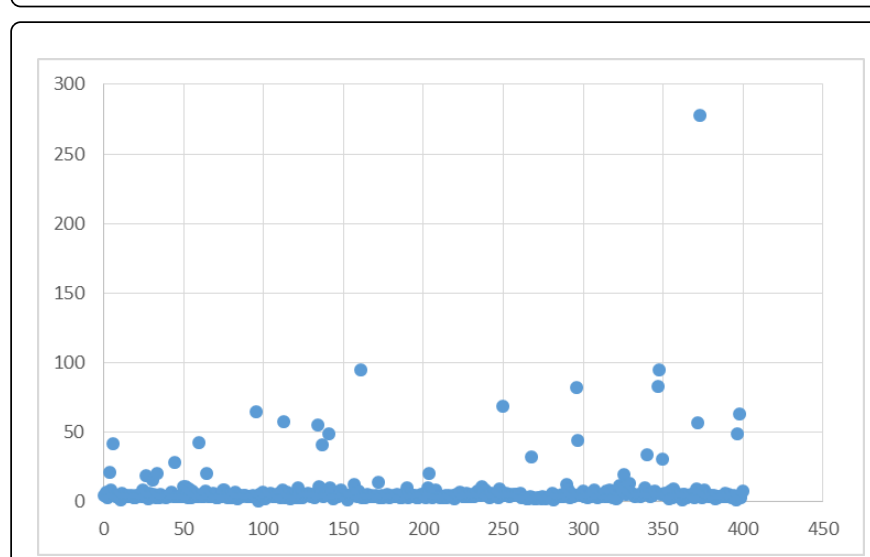

Figure 5: Number of miscellaneous bouts (in seconds) performed during a competitive surfing heat (average \pm standard deviation).

Figures 6 to 10 show the percentage intervals (in seconds) for each activity of all surfers during the heat.

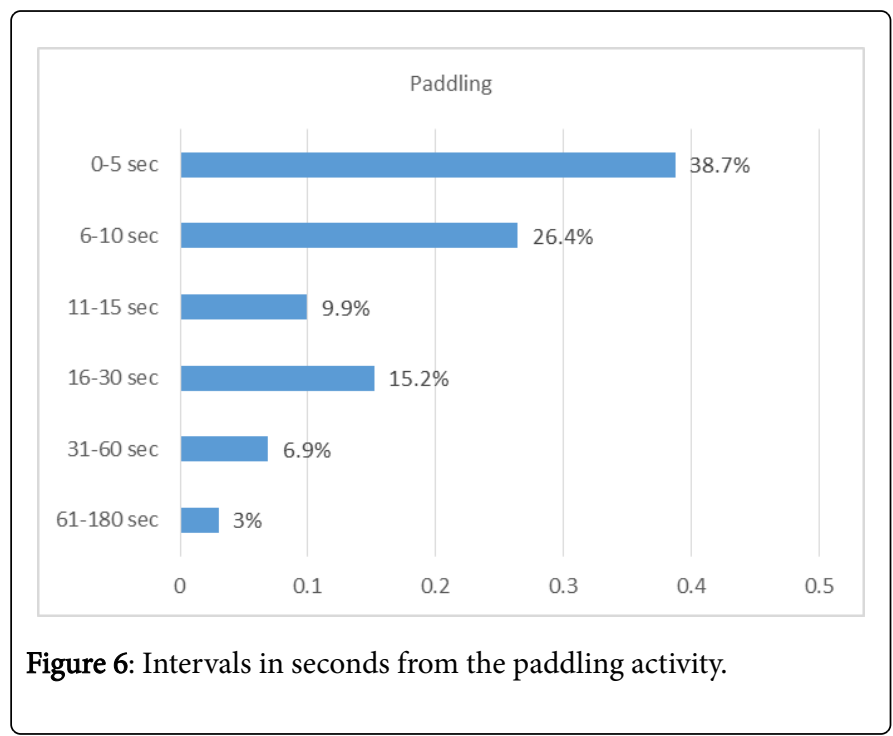




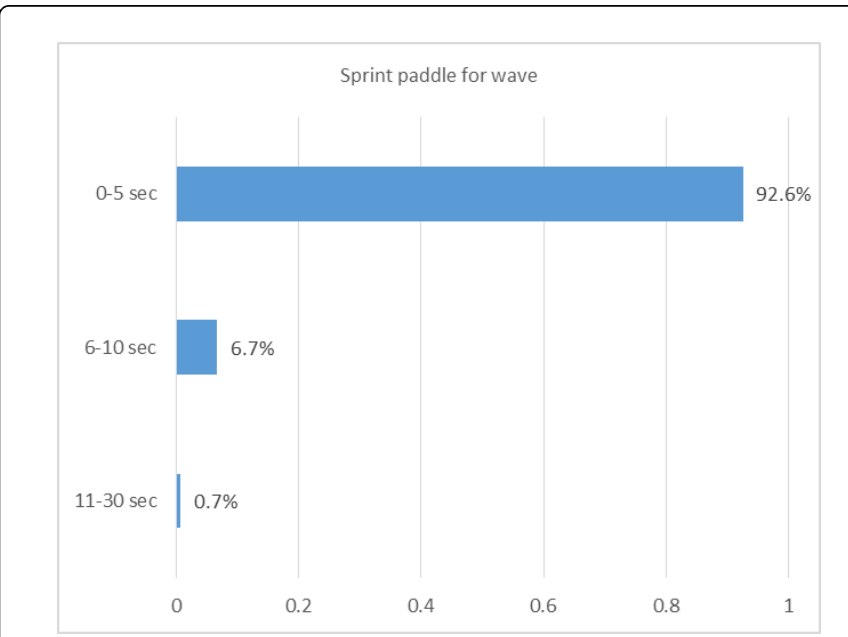

Figure 7: Intervals in seconds from the sprint paddle for wave activity.

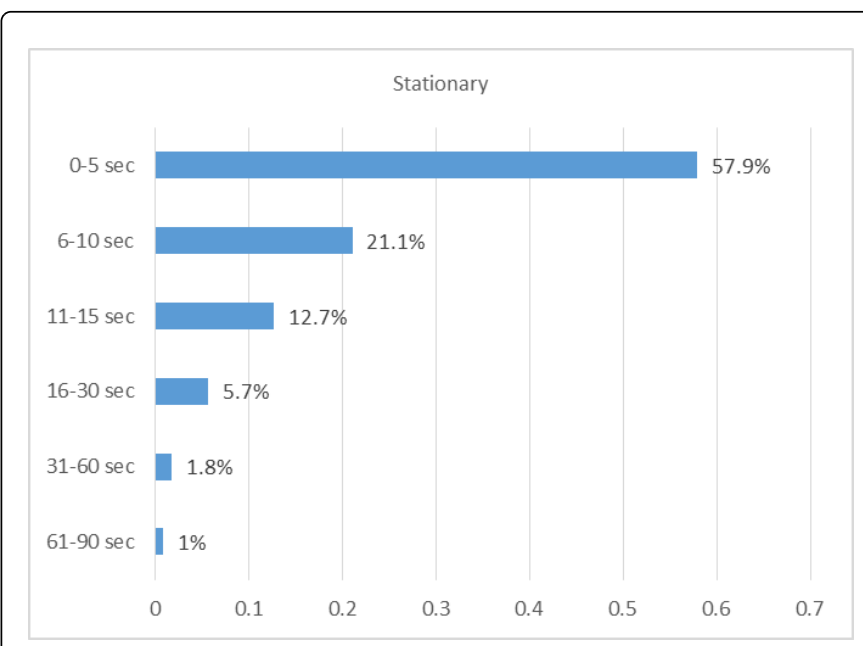

Figure 8: Intervals in seconds from the stationary period.

\section{Discussion}

To our knowledge, this is the first study that investigating the timemotion analysis of young surfers during competition.

The data of this study revealed that the paddling was the most practiced activity in surf (58\%), as already observed in other studies $[1,6,9,10]$, however, the great percentage of this movement was short duration (up to 5 seconds) and the longest paddling movement (between 61 and 180 seconds) had a small percentage, suggesting an activity with intercalary movements involving anaerobic metabolism, where the resynthesize of ATP is done through chemical reactions that do not require the presence of oxygen [11]. The data from the study by Mendez-Villanueva et al. [1] verified that most of the intervals of the paddling corresponded to a longer period (between 11 and 20 seconds), but the proximate intervals more executed were done up to 10 seconds.

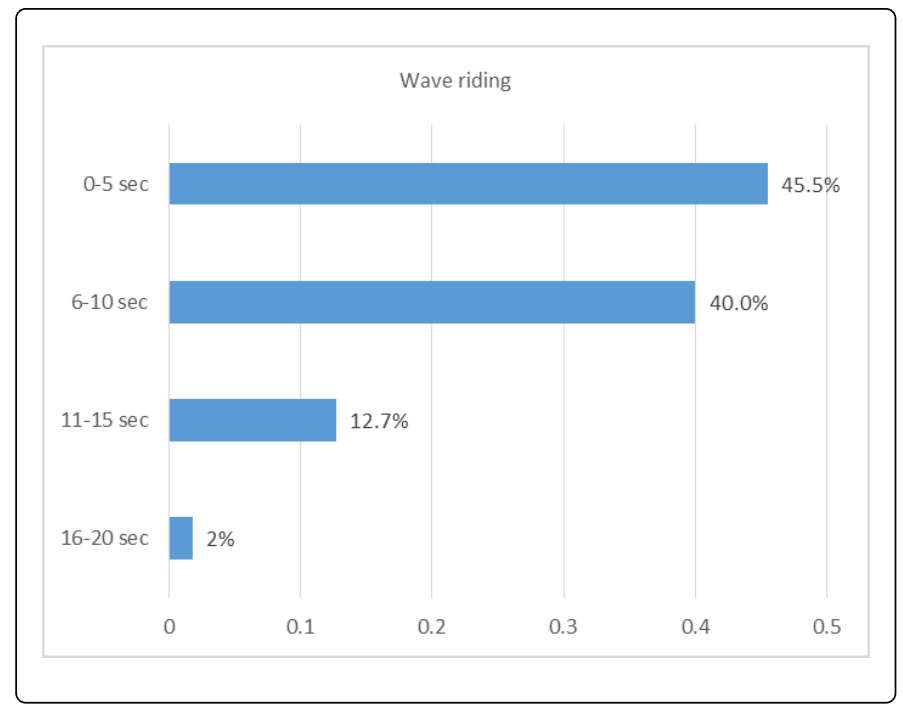

Figure 9: Intervals in seconds from the wave riding activity.

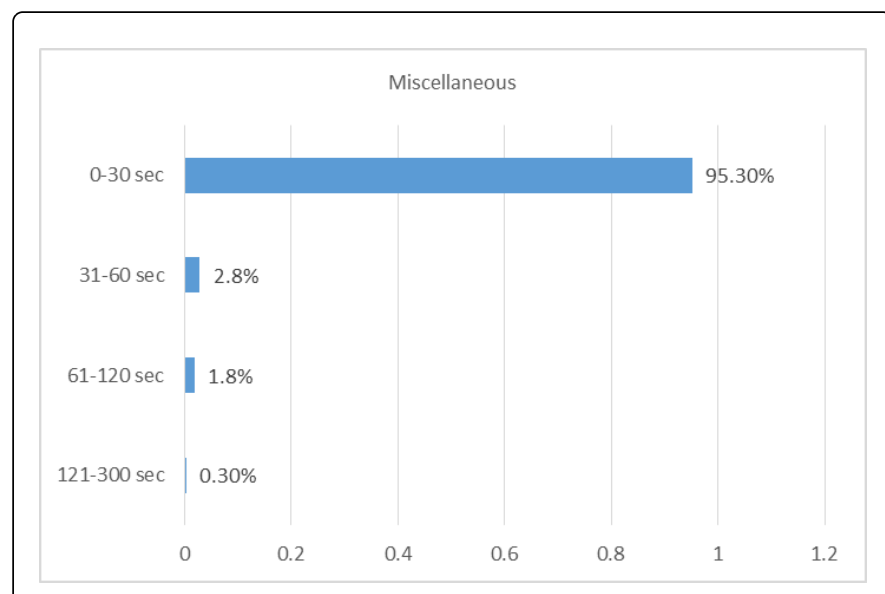

Figure 10: Intervals in seconds from miscellaneous.

The majority of the movement intervals of sprint paddle to catch the wave were also of short duration (up to 5 seconds), thus we can conclude that for the execution of this activity the the same anaerobic metabolism is requested, phosphatic system, because it involves fast and vigorous movements [11].

The total time to catch wave was only $3 \%$. Meir et al. reported that $5 \%$ of the total time was spent wave riding during 1 hour of recreational surfing, Secomb et al. revealed $2.5 \%$ in 2-h surfing training session, Mendez-Villanueva et al. showed results of $3.8 \%$ in competitive surfers, and Farley et al. reported $8 \%$ of wave riding in surfers from the current top 30 ranked surfers in New Zealand. This difference can be associated to the different conditions evaluated (free-surf and competition), since the athlete has to select the best waves to obtain the highest score, and if the athlete spends his time catching many waves without much quality, would lose his time paddling to return to the peak, and this time be accounted in the period of the heat; an athlete during the training or free-surf doesn't have this type of concern, being able to catch a greater amount of waves, without being selecting or waiting for the best wave set to surf. During competition the surfers 
were judged for the best 2 scored waves, not by the greatest amount of surf waves. In addition, the competitive level of athletes of these studies sample may also influence the results obtained, since in the mentioned studies the sample consisted of elite athletes and the sample analyzed in this study included young regional athletes.

The average length of a wave ride in the present study was 4.4 seconds; different data were obtained by Mendez-Villanueva et al. which revealed 11.6 seconds of average value. It's difficult to compare our results with those obtained in the last study since they are different samples (this study involved children and adolescents and in the Mendez-Villanueva et al. study the sample consisted of elite-level, professional surfers ranked in the World Qualifying Series (WQS). Besides that, these disagreements simply reflect the differences between the lengths of the waves in the 2 surfing venues, because different locations revealed different surf conditions and lengths waves that will allow surfers longer or small rides. As well as different tides, wave break, wind directions also provide different swell.

By analyzing the time interval of each activity mentioned above it can be verified that there is little disintegration of the glycogen in lactic acid, since this type of metabolism is present in activities with duration between 1 and 3 minutes; lactic acid is a co-product of anaerobic glycolysis and when it builds up at high levels in muscles and blood it causes muscle fatigue [11].

The surfers in this competition were more active, because the total stationary time being only $13 \%$, different with values obtained in the studies of Secomb et al. (53\%), Meir et al. (44\%), Mendez-Villanueva et al. (42\%), and Farley et al. (28\%). The stationary time is dependent on several variables; among them the motivation of the athlete and the period between waves.

The intervals of stationary period in this study were very short (up to 5 seconds), revealing that the athlete needs only a brief recovery period and was always looking for the best sea conditions moving during the heat. Mendez-Villanueva et al. revealed that the majority of surfers performed intervals of up to 10 seconds, followed by intervals between 11 and 20 seconds.

The time devoted to miscellaneous (e.g., duck diving, recovering the surfboard) accounted for $23 \%$ of the total heat time in the current study. This value is clearly higher than the $16 \%$ reported by Meir et al. and the $2.2 \%$ by Mendez-Villanueva et al. These variations can be explained by the fact that some of the surfers chose to leave the water and return in the place where the entrance was facilitated by the direction of the current, and this exit of the water was also included in the other activities in this study, reflecting methodological differences between the studies.

The percentage of intervals of miscellaneous activities also revealed a greater dynamism of the athletes, since the great majority of the surfers was involved with these activities in a maximum of 30 seconds, performing hereafter another type of activity.

Data from this study can be used as a reference value to adapt training programs to the temporal work demands of surfing competition and may prove useful for specific conditioning and to develop appropriate training protocols. However, the data obtained only provide information about that population studied, in that particular place and with the specific conditions of the day of the evaluation, since the surf conditions and type of wave breaks influenced the durations of time and per cent of time the surfers spent performing each respective activity.

It is necessary to realize more studies at national level, involving elite athletes, both adults and young surfers, for a better analysis of the total time of surfing activity.

\section{Conclusion}

The data from this study revealed that, for this sample and competition analyzed, the activities that had a greater percentage of time included the paddling and miscellaneous activities.

The results of this study show that surfing is an intermittent activity that is characterized by large variability of each activity that was analyzed, and these surfing activities seems to be controlled more by environmental factors than by surfer attitude.

\section{Acknowledgement}

Desporto Escolar Organization; José Lourenço; Luís Reis.

\section{Conflict of Interest}

None.

\section{Funding}

Research in Education and Community Intervention (RECI); Foundation for Science and Technology (FCT); Reference Framework National strategic (QREN); COMPETE; European Union

\section{References}

1. Mendez-Villanueva A, Bishop D, Hamer P (2006) Activity profile of world-class professional surfers during competition: a case study. J Strength Cond Res 20: 477-482.

2. https://www.isasurf.org/. Accessed May 23, 2017

3. Redd M, Fukuda D (2016) Utilization of time motion analysis in the development of training programs for surfing athletes. Strength Cond J 38: $1-8$.

4. https://www.surfingportugal.com/cms/index/view/gid/9917

5. Moraes G, Guimarães A, Gomes A (2013) Analysis of the prevalence of injuries in the Paraná coast surfers. Acta Ortop Bras 21: 213-218.

6. Farley O, Harris N, Kilding A (2012) Physiological demands of competitive surfing. J Strength Cond Res 26: 1887-1896.

7. Mendez-Villanueva A, Bishop D (2005) Physiological aspects of surfboard riding performance. Sports Med 35: 55-70.

8. Farley O (2011) Competitive surfing: A physiological profile of athletes and determinants of performance. Master's Thesis, Auckland University of Technology.

9. Secomb J, Sheppard J, Dascombe B (2015) Time-motion analysis of a 2hour surfing training session. Int J Sports Physiol Perform 10: 17-22.

10. Meir R, Lowdon B, Davie A (1991) Heart rates and estimated energy expenditure during recreational surfing. J Sci Med Sport 23: 70-74.

11. McArdle W, Katch F, Katch V (2015) Exercise physiology: Nutrition, energy, and human performance (8th edn.). Wolters Kluwer Health, Baltimore, USA. 